\title{
Aferição de uma escala de atitudes para avaliação da satisfação no trabalho
}

\author{
J. CORREIA JESUINO* \\ L. SOCZKA** \\ J. GUERREIRO MATOSO ***
}

\section{INTRODUÇÃO}

Existem numerosos estudos sobre atitudes dos trabalhadores para com a sua própria empresa ou organização destinados a avaliar o grau de satisfação ou, como por vezes também se designa, o moral. Tais estudos têm sido levados a efeito sobretudo nos Estados Unidos, no âmbito do «comportamento organizacional», daf provindo os prin. cipais métodos e técnicas utilizados. O principal objectivo da avaliação do moral dos trabalhadores baseava-se, inicialmente, no pressuposto de que, quanto maior a satisfaçáo dos trabalhadores pelo seu próprio trabalho maior seria a produtividade.

A evidência empírica reunida veio, porém, a revelar uma relação mais complexa e indirecta entre satisfação e produtividade e daí que, actualmente, a tendência seja para avaliar, não um

\footnotetext{
- Docente de Psicologia Social no ISCTE e no ISPA

** Psicólogo Social no Centro de Informática, LNEC

*** Membro do Conselho Directivo do Instituto Nacional de Estatística
}

índice global de satisfação para com o trabalho mas as diferentes dimensōes que a integram.

Muito provavelmente a atitude para com o trabalho é multifacetada e daí os resultados contraditórios que se encontram quando se seleccio. nam índices globais com critérios como a produ. tividade, o absentismo ou a rotatividade.

Esta diferente filosofia está subjacente à metodologia desenvolvida por Pat Smith e colaborado. res (1969) e conhecida sob a designaçăo de Indice Descritivo do Trabalho (Job Descriptive Index JDI). 0 questionário é dividido em várias secçōes, cada uma das quais consistindo numa escala com. preendendo uma série de objectivos descritivos. Os vários aspectos abordados são, habitualmente, as condiçōes de trabalho, salário, perspectivas de promoçăo, supervisão e colegas. As escalas são Tricotómicas, respectivamente S (Sim) quando o respondente está de acordo, $\mathrm{N}$ (Não) quando está em desacordo e ? (Não sei) quando năo sabe qual a melhor escolha,

A vantagem desta técnica reside, em primeiro lugar, na oportunidade que fornece de determinar - grau de satisfação ou de insatisfaçăo $\mathrm{em}$ cinco diferentes áreas ocupacionais, e portanto quais os 
factores que proporcionam maior ou menor satisfação. Um outro aspecto que torna recomendável a técnica de Smith e colaboradores é a facilidade de administração da escala, tornando desnecessária a sua adaptação para cada ocupação específica. Ela permite efectuar comparações entre diferentes organizações ou diferentes secções da mesma organização.

Não obstante as vantagens enumeradas o JDI, concebido essencialmente para avaliar as atitudes dos estratos ocupacionais mais baixos de empresas norte-americanas, levanta problemas quanto à sua aplicação a outros estratos profissionais e noutros contextos culturais. Para obviar a tais inconvenientes, Warr e Routledge (1969) elaboraram uma escala baseada nos mesmos princípios do JDI mas adaptada a uma "cultura de empresa» mais europeia e susceptível de ser aplicada não apenas a executantes mas também a quadros com funções de supervisão ou chefia.

A escala proposta inclui, assim, subsecções relativas a perspectivas de promoção, conteúdo de trabalho, superior imediato, colegas do mesmo nível, subordinados e organização como um todo. A estrutura é, pois, semelhante ao JDI, mas melhor adaptada ao contexto visado. No que se refere à redacção das questões a incluir nas diferentes subsecções os autores procuraram obedecer aos seguintes princípios:

a) Eliminação das questões mais relacionadas com o contexto do trabalho tais como ruído, sujidade, calor, etc. por se afigurarem menos pertinentes para o estrato ocupacional visado;

b) Tornar as questões relevantes para amostras britênicas. Por exemplo indagar sobre os princípios da filosofia cooperativa não tem sentido para os respondentes ingleses;

c) As questões serem duma natureza bastante geral, relevantes em diferentes organizações e empresas;

d) Se possível redutíveis a um adjectivo ou a uma frase curta;

e) Não serem apenas sinónimos ou antónimos de questões já incluídas; f) Aproximadamente metade das questões em cada subsecção serem positivas e as restantes serem negativas.

Uma vez constituída a escala, Warr e Routledge (1969) procederam ao estudo da sua normalização mediante diferentes aplicações a amostras de diferentes empresas industriais. A primeira dessas aplicações, administrada e uma amostra de 200 sujeitos, levou à redução das questões inicialmente incluídas nas diferentes subescalas, por forma a aumentar a sua validade interna.

$\mathrm{Na}$ sua forma resultante, a escala de Warr e Routledge (1969) compreende um total de 77 questões divididas por 7 subescaląs, com coeficientes de validade, calculados pelo método «half-split», compreendidos entre .74 e .86 .

No que se refere à validade de construto Warr e Routledge (1969) recorreram ao método «multitraço-multimétodo» (Campbell e Fiske, 1959) utilizando para o efeito, para além das 7 subescalas descritas, outras 7 subescalas tipo Lickert de 9 pontos, relativos à apreciação global de cada uma das subsecções.

A partir dos dois índices assim obtidos para cada subsecção calcularam a matriz de intercorrelações simples com base na qual se verifica a validade convergente e discriminante do inquérito.

Tendo em conta os resultados obtidos por Warr e Routledge (1969) e considerando por outro lado, por simples inspecção visual, que o conteúdo das questões se afigurava satisfatório para utilização em Portugal, resolvemos proceder à sua adaptação para português procurando igualmente determinar as suas propriedades psicométricas. A ideia fundamental que presidiu no trabalho efectuado consistiu em preparar um instrumento de medida simples e robusto que permitisse servir de base para os estudos de satisfação no trabalho e que seja útil tanto para futura investigação no domínio do comportamento organizacional como para fins de diagnóstico expedito do moral das organizações em empresas.

\section{MÉTODO}

\section{Instrumento}

Fscala Warr e Routledge (1969) na sua versão em português. A escala é composta por 7 subescalas apresentadas na seguinte ordem: (entre parên- 
tesis indica-se o número de questర̄es) organização (12), remuneraçōes (7), superior imediato (13), colegas do mesmo nível que o seu (10), perspec. tivas de promoçẵo (8), trabalho em si mesmo (15), subordinados (11). Cada questẫo de cada subescala é precedida de três alternativas designadas por $\mathrm{S}$ - correspondente a Sim, N-correspondente a Não e ? - correspondente a Não sei. O respondente assinala a alternativa que melhor corresponde à sua resposta. A cotação é de 3 para $S$ quando a questão é positiva (por exemplo: boa reputaçăo) e 3 para $\mathrm{N}$ quando negativa (p. ex. coordenação insuficiente entre departamentos), 1 para $\mathrm{S}$ quando negativa, 1 para $\mathrm{N}$ quando negativa, e 2 para ?. O score em cada subescala é determinado somando as pontuaçōes ao longo das questōes que a compōem.

Em cada secção, para além destas subescalas inclui-se ainda uma segunda subescala com um única questăo, com 9 pontos, sob forma de diferen. ciador semântico e que se destinava a recolher a opiniăo global do respondente sobre o tópico a que a seç̧ão diz respeito.

Em Anexo reproduz-se o questionário na sua totalidade com as respectivas subescalas.

\section{Sujeitos}

$\mathrm{O}$ questionário foi aplicado em duas unidades geograficamente distintas aqui designadas por $\mathrm{L}$ e $\mathrm{T}$ - do Centro de Informática de um organismo de
Administração Pública, às várias categorias profissionais, tais como: chefias, analistas e programadores, operadores de registo de dados e outras (pessoal de enquadramento administrativo, técni$\cos$ auxiliares, preparadores e arquivistas de suporte). 0 questionário foi passado a todo o pessoal disponível em cada uma das unidades, respectivamente a 125 sujeitos em L e a 108 sujeitos em T. Vieram todavia a registar-se $31 \%$ de quebras em L e $37 \%$ em $\mathrm{T}$ por virtude de preenchimento insuficiente dos protocolos, pelo que a amostra ficou reduzida a 86 sujeitos em L e 68 sujeitos em T.

A unidade $T$ era bastante recente, a data do questionário, e a generalidade do pessoal estava na situação de contrato a prazo.

\section{RESULTADOS}

\section{Análise psicométrica}

\section{Validade interna}

Apurados os resultados procedeu-se em primeiro lugar a uma análise psicométrica das subescalas do inquérito para cada amostra, de $\mathrm{L}$ e de $\mathrm{T}$.

Para o efeito procedeu-se ao cálculo das correlaçōes item-teste para cada subescala e respectivo coeficiente Alfa de Cronbach (1951) e que permite uma avaliação da validade interna das escalas.

No Quadro I, coluna (1), são reproduzidos os valores de Alfa para seis das sete subescalas do

Quadro I - Coeficientes Alfa de Cronbach

\begin{tabular}{|c|c|c|}
\hline & L & $\mathrm{T}$ \\
\hline Subescala & (1) (2) & (1) (2) \\
\hline I. Organizaçẫo $\ldots \ldots \ldots \ldots \ldots$ & $.608 / .741$ & $.403 / .601$ \\
\hline II. Remuneraçôes $\ldots \ldots \ldots \ldots \ldots \ldots$ & $.554 / .487$ & $.628 / .662$ \\
\hline III. Superior Imediato $\ldots \ldots \ldots \ldots \ldots$ & $.945 / .945$ & $.835 / .835$ \\
\hline IV. Colegas do mesmo nível . . . . . . . & $.796 / .808$ & $.708 / .740$ \\
\hline V. Perspectivas de promoção ....... & $.782 / .782$ & $.695 / .695$ \\
\hline VI. Trabalho em si mesmo ......... & $.870 / .883$ & $.645 / .702$ \\
\hline
\end{tabular}




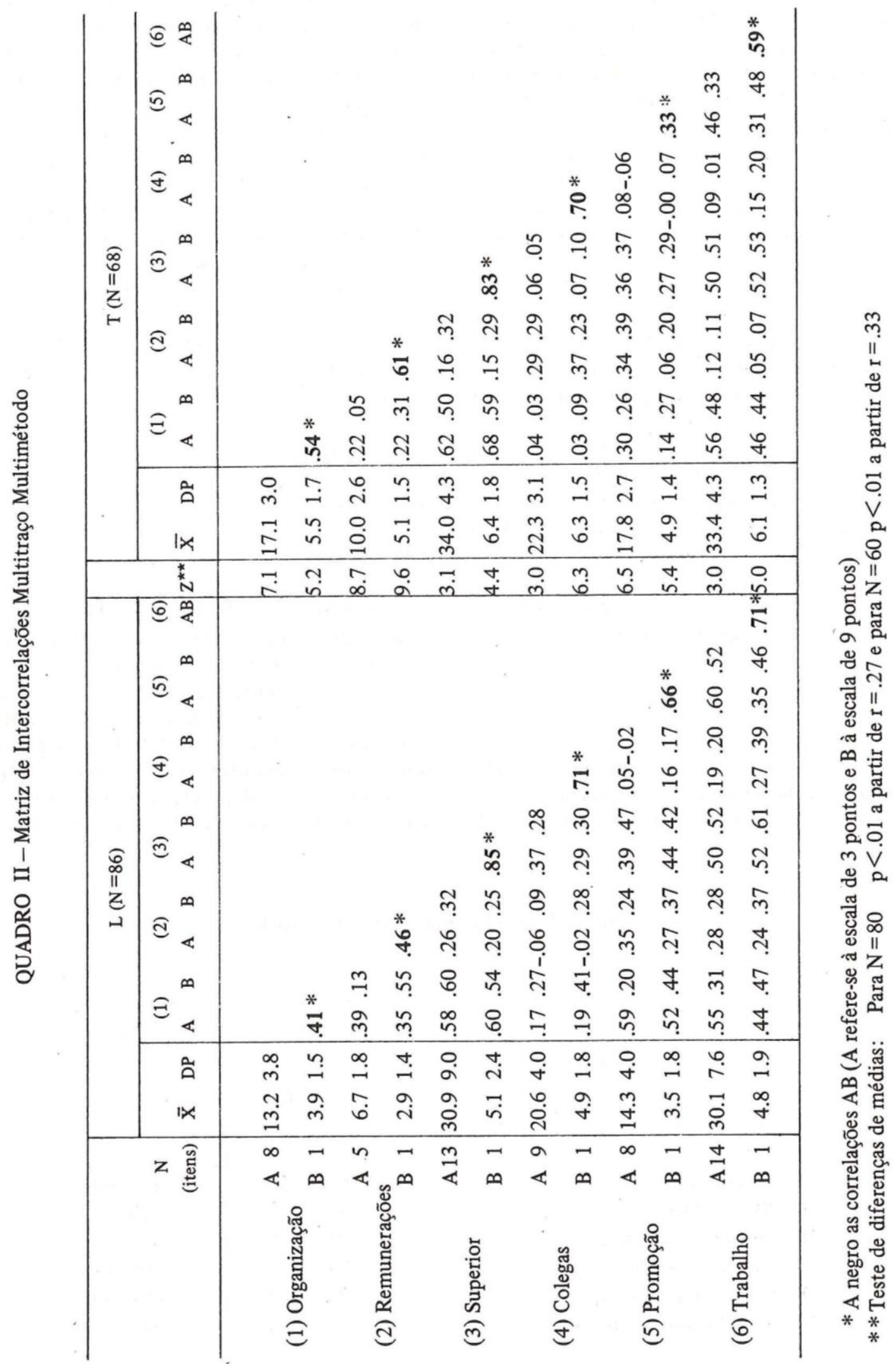


questionário, para as unidades L T. Não se procedeu ao cálculo do coeficiente Alfa para a subes. cala sete, relativa aos subordinados, por virtude do reduzido número de respondentes com funçø̄es de chefia que preencheram a subescala $-28 \mathrm{em}$ Le $8 \mathrm{em} \mathrm{T}$.

No que se refere às correlaçốes item-teste para cada subescala verificou-se que alguns dos itens apresentavam correlaçōes muito baixas pelo que se procedeu à sua eliminaçắo. Assim, na subesca. la I foram eliminados os itens $1,2,4$ e 9 , na subescala II foram eliminados os itens 3 e 5 , na subescala IV foi eliminado o item 10 e na subescala VI foi eliminado o item 15.

Uma vez eliminados estes itens voltou a proce. der-se ao cálculo dos coeficientes Alfa. Os resul. tados obtidos constam da coluna (2) do Quadro I. As correlaçōes item-teste săo indicadas entre parêntesis para cada item retido no questionário reproduzido em Anexo.

A média dos coeficientes Alfa é de .77 para a amostra $\mathrm{L}$ e de .71 para a amostra $\mathrm{T}$, o que indica serem os dados de $\mathrm{L}$ de maior confiança do que os dados de T. Quanto à validade relativa das subescalas verifica-se que a subescala II respeitante a remuneraçōes oferece pouca confiança para ambas as amostras e que o mesmo se aplica à subescala I para a amostra T. O baixo coeficiente Alfa obtido para a escala II mostra com efeito grande variabilidade das respostas sendo de pôr em dúvida a sua unidimensionalidade. As mesmas consideraçōes șio aplicáveis à escala I para a amostra $\mathrm{T}$.

\section{Validade convergente e discriminante}

Uma vez determinada a validade interna das escalas procedeu-se em seguida ao exame da validade convergente e discriminante recorrendo ao método multitraço-multimétodo de Campbell e Fiske (1959). Segundo estes autores o método é aplicável quando se examinam ao mesmo tempo mais do que um so traço (no caso vertente num factor de satisfação) por mais do que um método.

A validade convergente requer que os diferentes métodos de medida do mesmo traço (ou factor de satisfação) tenham intercorrelaçōes elevadas. A validade discriminante requer que as medidas dos traços (ou factores de satisfaçăo) não estejam de facto correlacionados entre si. A validade discriminante é função de duas condiçōes: em primeiro lugar se a correlaçāo entre o índice da subescala de 3 pontos e o índice da subescala de 9 pontos for superior às correlaçōes entre esses dois índices ou entre os índices de todas as restantes subescalas; e, em segundo lugar, se a correlaçẵo entre os indices das duas subescalas para determinado tópico for superior às correlaçóes do índice da subescala de 3 pontos com todos os índices das mesmas subescalas em todos os outros tópicos.

Os resultados correlacionais obtidos separadamente para a amostra $\mathrm{L}$ e para a amostra $\mathrm{T}$ estão reproduzidos no Quadro II.

As colunas e linhas designadas por $A$ dizem respeito aos dados das subescalas de 3 pontos somando os diversos itens de cada subescala. As colunas e linhas designadas por $B$ dizem respeito ao juízo enunciado com o diferenciador semântico de 9 pontos, incluído a seguir a cada subescala.

Para demonstrar a validade convergente é necessário que as correlaçб̄es $\mathrm{AB}$, que figuram o sublinhado na diagonal do Quadro II, sejam significativas, condição essa que se verifica plenamente. Quanto ao requisito da validade discriminante é necessário que as correlaçôes $\mathrm{AB}$ para cada uma das seç̧čes (1) a (6) sejam superiores às restantes correlaçōes $\mathrm{AB}$ e AA ao longo da respectiva linha $\mathrm{e}$ da respectiva coluna.

Tal como se pode verificar a partir do Quadro II, esta segunda condiçắo não se verifica para as escalas I e II na amostra L, e para as escalas I e V na amostra T. Isso significa que a escala $I$ năo é suficientemente discriminativa relativamente às restantes escalas reflectindo-se nos seus scores factores provenientes das outras escalas. Isto em ambas as amostras. Por outro lado, em L, as mesmas consideraçốes são aplicáveis à escala II (remuneraçōes) em $\mathrm{T}$ à escala $\mathrm{V}$ (perspectivas de promoção).

Efectuou-se em seguida o cálculo para a populaçăo total, ou seja, para as duas amostras em conjunto. Os resultados constam do Quadro III.

Os resultados obtidos para a população total confirmam a validade convergente e discriminante da escala com excepção da escala I, relativa à apreciação como um todo, e que requer pois a reformulação por forma a torná-la independente das restantes escalas que constituem o questionário. Para além disso torna-se igualmente necessário proceder a ajustamentos na escala das remuneraçóes e das perspectivas de promoção dado que sujeitas a variaçăo em função das amostras a que é 
(1) (2) (3)

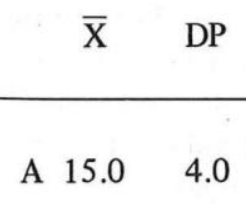

(1)

A B

A

(3)

(4)

(5)

(6)

$\begin{array}{lllll}\text { B } & 4.6 & 1.8 & .58 * & \\ \text { A } & 8.2 & 2.7 & .50 & .35\end{array}$

(2)

$\begin{array}{llllll}\text { B } & 3.9 & 1.8 & .50 & .59 & .71\end{array}$

$\begin{array}{lllllll}\text { A } & 32.3 & 6.8 & .61 & .57 & .30 & .38\end{array}$

(3)

$\begin{array}{rrrrrrrrr}\text { B } & 5.7 & 2.2 & .67 & .60 & .30 & .37 & .84 & \\ \text { A } & 21.4 & 3.8 & .23 & .26 & .22 & .28 & .33 & .26\end{array}$

(4)

$\begin{array}{rrrrrrrrrrr}\text { B } & 5.5 & 1.8 & .29 & .39 & .34 & .39 & .29 & .31 & .72 & \\ \text { A } & 15.9 & 3.9 & .61 & .38 & .50 & .47 & .43 & .50 & .16 & .13\end{array}$

(5)

$\begin{array}{lrlllllllllll}\text { В } & 4.1 & 1.8 & .50 & .18 & .35 & .45 & .44 & .44 & .19 & .25 & .63 & \\ \text { A } & 31.5 & 6.5 & .58 & .42 & .30 & .31 & .53 & .54 & .22 & .22 & .60 & .54\end{array}$

(6)

$\begin{array}{llllllllllllll}\text { В } & 5.4 & 1.8 & .54 & .54 & .33 & .41 & .55 & .62 & .30 & .42 & .44 & .54 & .71\end{array}$

* A negro as correlações $\mathrm{AB}$ (A refere-se à escala de 3 pontos e $\mathrm{B}$ à escala de 9 pontos. Para $\mathrm{N}=150$ $\mathrm{p}<.01$ a partir de $\mathrm{r}=.21$.

aplicada. Quanto às escalas relativas ao superior imediato, aos colegas e ao conteúdo de trabalho, verifica-se que são instrumentos de elevada confiança.

\section{Análise factorial}

Procedeu-se em seguida a uma análise factorial das escalas tanto em relação às duas amostras em conjunto como para cada amostra separadamente. Utilizou-se para o efeito o método das componentes principais com rotação Varimax. Os resultados obtidos são reproduzidos no Quadro IV.

Os resultados mostram claramente a existência dum primeiro factor explicando perto de $50 \%$ da variação total e para a qual concorrem as atitudes expressas para com a organização como um todo, para com o superior imediato, perspectivas de promoção e conteúdo de trabalho, um segundo factor respeitante às remunerações. No caso da amostra $T$ a análise revela a existência dum quarto factor respeitante a perspectivas de promoção. A análise factorial confirma deste modo os dados obtidos mediante a análise discriminante e sugere que a atitude expressa sobre a organização como um todo é em grande parte determinada pela atitude para com os superiores imediatos e para com o conteúdo do trabalho.

\section{Análise de correlação/regressão múltipla}

Procedeu-se em seguida a uma análise de correlação/regressão múltipla em primeiro lugar para o conjunto das escalas como um todo e em seguida para cada uma das subescalas, e isso tanto para a 


\begin{tabular}{|c|c|c|c|c|c|c|c|c|c|c|c|}
\hline \multirow{2}{*}{ Escalas } & & \multicolumn{3}{|c|}{$\mathrm{L}+\mathrm{T}(154)$} & \multicolumn{3}{|c|}{ L (86) } & \multicolumn{4}{|c|}{$\mathrm{T}(68)$} \\
\hline & & I & II & III & I & II & III & I & II & III & IV \\
\hline & 1 & .69 & .09 & -.45 & .74 & .11 & .28 & .85 & -.01 & -.44 & -.00 \\
\hline \multicolumn{12}{|l|}{ Organização } \\
\hline & 2 & .58 & .29 & -.36 & .31 & .54 & .52 & .73 & .02 & -.08 & .16 \\
\hline & 3 & .13 & .17 & -.87 & .29 & -.21 & .68 & .06 & .28 & -.82 & -.03 \\
\hline \multicolumn{12}{|c|}{ Remunerações } \\
\hline & 4 & .23 & .26 & -.82 & .15 & .18 & .88 & .19 & .16 & -.84 & .04 \\
\hline & 5 & .82 & .23 & -.08 & .66 & .44 & .21 & .83 & .01 & -.17 & .14 \\
\hline \multicolumn{12}{|l|}{ Superior } \\
\hline & 6 & .86 & .19 & -.11 & .74 & .39 & .10 & .88 & .02 & -.14 & .18 \\
\hline & 7 & 16 & .87 & -.08 & .12 & .82 & -.11 & .01 & .87 & -.48 & .03 \\
\hline \multicolumn{12}{|l|}{ Colegas } \\
\hline & 8 & .17 & .89 & -.21 & .05 & .87 & .09 & .04 & .92 & -.12 & .07 \\
\hline & 9 & .58 & -.13 & -.58 & .83 & -.19 & .11 & .25 & -.18 & -.58 & .50 \\
\hline \multicolumn{12}{|l|}{ Promoção } \\
\hline & 10 & .59 & .01 & -.45 & .70 & .60 & .28 & .10 & .00 & -.09 & .87 \\
\hline & 11 & .78 & .02 & -.20 & .81 & .13 & .09 & .62 & .01 & -.04 & .48 \\
\hline \multicolumn{12}{|l|}{ Trabalho } \\
\hline & 12 & .75 & .26 & -.18 & .63 & .39 & .22 & .57 & .24 & .14 & .61 \\
\hline
\end{tabular}

$$
\begin{aligned}
\mathrm{L}+\mathrm{T} & -\% \text { acumulada I (.49) II }(.65) \mathrm{III}(.71) \\
\mathrm{L} & -\% \text { acumulada I (.44) II }(.59) \mathrm{III}(.68) \\
\mathrm{T} & -\% \text { acumulada I }(.37) \mathrm{II}(.55) \mathrm{III}(.65) \mathrm{IV}(.74)
\end{aligned}
$$

amostra de Lisboa como para a amostra de Tomar. Em ambos os casos a análise de regressão foi efectuada tomando cada uma das subescalas sucessivamente como variável dependente.

A ideia subjacente a estas sucessivas análises consistia em determinar em primeiro lugar a importância relativa das subescalas enquanto factor explicativo das outras e, em segundo lugar, determinar o peso relativo dentro de cada subescala das questões nelas incluídas. Vamos proceder à descrição separada dos resultados obtidos em cada um dos casos.

\section{Interescalas}

No Quadro V indicam-se os resultados obtidos tomando sucessivamente como variável dependente (VD) cada uma das subescalas e regredindo relativamente a ela as restantes escalas. Para o efeito utilizaram-se as subescalas tricotómicas.

Recorreu-se ao método "passo a passo» (stepwise), que consiste na optimização da regressão por busca automática e sem obediência a qualquer hierarquização prévia. Este método é sobretudo recomendável quando não se dispõe duma hipótese teórica sobre o grau de importância relativa das variáveis independentes (Cohen e Corhn 1975).

Atendendo a que os resultados de L são significativamente inferiores, em todas as escalas, aos resultados de $\mathrm{T}$ podemos então interpretar em termos globais os resultados da regressãó múltipla nos seguintes termos. 
Quadro V-Percentagens de variância explicada $\left(\mathrm{R}^{2}\right)$

para as subescalas em conjunto

\begin{tabular}{|c|c|c|c|c|c|c|c|c|c|c|c|c|}
\hline \multirow{3}{*}{$\begin{array}{c}\text { Variáveis } \\
\text { Independentes }\end{array}$} & \multicolumn{12}{|c|}{ Variáveis Dependentes } \\
\hline & \multicolumn{2}{|c|}{ I } & \multicolumn{2}{|c|}{ II } & \multicolumn{2}{|c|}{ III } & \multicolumn{2}{|c|}{ IV } & \multicolumn{2}{|c|}{ V } & \multicolumn{2}{|c|}{ VI } \\
\hline & $\mathrm{L}$ & $\mathrm{T}$ & $\mathrm{L}$ & $\mathrm{T}$ & $\mathrm{L}$ & $\mathrm{T}$ & $\overline{\mathrm{L}}$ & $\mathrm{T}$ & $\mathrm{L}$ & $\mathrm{T}$ & $\mathrm{L}$ & $\bar{T}$ \\
\hline & & & & & & & & & & & & \\
\hline I (Organização) & - & - & .14 & - & .33 & .38 & - & - & .10 & - & - & .31 \\
\hline II (Remunerações & - & - & - & - & - & - & - & $.08 *$ & - & .08 & - & - \\
\hline III (Superior) & .15 & .38 & - & - & - & - & .13 & - & - & - & .09 & - \\
\hline IV (Colegas) & - & - & - & $.07 *$ & $.07 *$ & - & - & - & - & - & - & - \\
\hline V (Promoção) & .34 & - & - & .12 & - & - & - & - & - & - & .34 & .10 \\
\hline VI (Trabalho) & - & $.08 * *$ & - & - & $.03 *$ & .04 & - & - & .35 & .21 & - & - \\
\hline Total & $49 \%$ & $46 \%$ & $14 \%$ & $19 \%$ & $43 \%$ & $42 \%$ & $13 \%$ & $8 \%$ & $45 \%$ & $29 \%$ & $43 \%$ & $41 \%$ \\
\hline
\end{tabular}

$* \mathrm{p}<.02 \quad * * \mathrm{p}<.003 \quad$ Todos os restantes $\mathrm{p}<.001$

A insatisfação global para com a organização, em L, acha-se sobretudo associada às perspectivas de promoção (34\%) apenas atenuada pela conduta dos superiores imediatos (15\%) e, por seu turno, as perspectivas de promoção estão associadas no tipo de trabalho (34\%).

Note-se que não se trata aqui de estabelecer relações de casualidade linear mas apenas o conjunto de factores inter-relacionados e que melhor explicam, no sentido estatístico do termo, a satisfação ou insatisfação relativa dos sujeitos perante os vários aspectos da organização.

Paralelamente, em T, a satisfação global acha-se sobretudo associada à conduta dos superiores imediatos (38\%) e, em segundo lugar, ao tipo de trabalho (8\%) e por seu turno o tipo de trabalho acha-se associado à organização (31\%) e às perspectivas de promoção (10\%).

\section{Intra-escalas}

Para um maior aprofundamento da análise procedeu-se em seguida ao exame das subescalas do questionário com maior poder explicativo, procurando determinar para cada uma delas a estrutura factorial das questões que se compõem e regredindo-os em seguida relativamente à subescala de controlo incluída no final de cada secçạ̃o do questionário, ou seja, à subescala tipo diferenciador se- mântico de 9 pontos que, deste modo foi adoptada como critério (variável dependente). As análises foram feitas separadamente para a amostra $\mathrm{L}$ e para a amostra $\mathrm{T}$.

\section{Subescala I (Organização)}

No Quadro VI indicam-se os resultados da análise factorial por componentes principais e rotação Varimax.

Enquanto que a estrutura factorial da amostra L é relativamente clara, podendo interpretar-se o primeiro factor como um factor geral de satisfação para com a organização associado sobretudo ao seu funcionamento interno e o segundo factor relativo à sua imagem externa, na amostra de Tomar a estrutura torna-se menos clara e mais difícil de interpretar.

Seguidamente, para determinar o peso relativo dos diversos itens do questionário, procedeu-se a uma análise de correlação/regressão múltipla tomando como variável dependente a subescala de controlo resumindo a atitude global para com a organização como um todo. Os resultados são indicados no Quadro VII. 
Quadro VI - Solução factorial após rotação Varimax

\begin{tabular}{|c|c|c|c|c|c|}
\hline \multirow{2}{*}{ Itens } & \multicolumn{2}{|c|}{$\mathrm{L}$} & \multicolumn{3}{|c|}{$\mathrm{T}$} \\
\hline & I & II & I & II & III \\
\hline (1) Reputação $\ldots \ldots \ldots \ldots \ldots \ldots \ldots$ & & .84 & & & .68 \\
\hline (2) Direcção $\ldots \ldots \ldots \ldots \ldots \ldots \ldots$ & .60 & & .66 & & \\
\hline (3) Secretismo $\ldots \ldots \ldots \ldots \ldots \ldots$ & .78 & & & .77 & \\
\hline (4) Eficiência. . . . . . . . . . & & .80 & & .82 & \\
\hline (5) Distâncias. . . . . . . . . . . & .80 & & .77 & & \\
\hline (6) Regras e Regulamentos.......... & .70 & & .77 & & \\
\hline (7) Coordenação. $\ldots \ldots \ldots \ldots \ldots \ldots$ & .67 & & & & .66 \\
\hline (8) Esforço Pessoal . . . . . . . . . & & .53 & & & .69 \\
\hline
\end{tabular}

$\mathrm{L}-\%$ acumulada I $(37,9 \%)$ II $(58,5 \%)$

$\mathrm{T}-\%$ acumulada I $(27,1 \%)$ II $(43,6 \%)$ III $(58,4 \%)$

Quadro VII - Correlação/Regressão Múltipla da Subescala I relativamente à Subescala de Controlo I (VD)

Variáveis

Independentes

Amostra L

\begin{tabular}{|c|c|c|c|c|c|c|c|c|c|c|}
\hline \multirow{2}{*}{\multicolumn{2}{|c|}{ Independentes }} & & & & & & & & & \\
\hline & & $\mathrm{R}^{2}$ & $\Delta \mathrm{R}^{2}$ & $\mathrm{p} *$ & Beta & se & B & se & $\mathrm{sr}^{2}$ & $\mathrm{pr}^{2}$ \\
\hline (1) & Reputação . . . . . . & .18 & & .000 & .44 & .09 & .82 & .17 & .20 & .27 \\
\hline (3) & Segredo........ & .26 & .08 & .002 & .30 & .09 & .69 & .22 & .09 & .12 \\
\hline & Constante . . . . . & 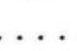 & $\cdots$ & $\ldots$ & $\cdots$ & $\ldots$ & 1.37 & .51 & & \\
\hline
\end{tabular}

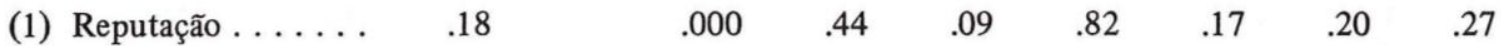

Amostra T

\begin{tabular}{lrrrrrrrr}
\hline $\mathrm{R}^{2}$ & \multicolumn{1}{l}{$\Delta \mathrm{R}^{2}$} & $\mathrm{p}{ }^{*}$ & \multicolumn{1}{l}{ Beta } & $\mathrm{se}$ & $\mathrm{B}$ & $\mathrm{se}$ & $\mathrm{sr}^{2}$ & $\mathrm{pr}^{2}$ \\
\hline & & & & & & & \\
.26 & & .000 & .51 & .096 & 1.20 & .23 & .26 & .43 \\
.39 & .13 & .000 & .36 & .096 & .66 & .18 & .13 & .22 \\
$\ldots \ldots$ & $\ldots \ldots$ & $\ldots \ldots$ & $\ldots \ldots$ & $\ldots$ & 1.38 & .70 & &
\end{tabular}

* - Significância do acréscimo de variância explicada

se - Erro padrão

sr - Coeficiente de correlação semiparcial

pr - Coeficiente de correlação parcial 
De acordo com os resultado obtidos verifica-se que a atitude negativa para com a organização na amostra de Lisboa se acha primariamente associada à reputação do serviço (18\%) e ao segredo que a Direç̧ão faz de assuntos que deveriam ser do conhecimento geral (12\%).

Em contrapartida em Tomar a atitude positiva para com a organização está primariamente associada à eficiência do serviço $(26 \%)$ e às regras e regulamentos adequados.

Os coeficientes Beta (estandardizados) dão indicação do peso relativo de cada uma das variáveis independentes na equação de regressão.

\section{Subescala III (Superior imediato)}

Tal como para o caso anterior efectuou-se em primeiro lugar uma análise factorial pelo método das componentes principais e rotação Varimax. Os resultados estão indicados no Quadro VIII.
$\mathrm{Na}$ amostra de $\mathrm{L}$ a estrutura factorial não é tão nítida como na de $T$. Nesta última o primeiro factor diz claramente respeito às relações de serviço entre chefe e subordinado, o segundo factor diz respeito às características pessoais $\mathrm{e}$ profissionais dos chefes.

Adoptando esta interpretação teríamos, relativamente à amostra de $\mathrm{L}$, um primeiro factor respeitante a qualidades profissionais e pessoais do chefe $(61 \%)$ e um segundo factor respeitante ao seu relacionamento específico em assuntos de serviço $(8 \%)$.

No que se refere à correlação/regressão múltipla os resultados obtidos são indicados no Quadro IX.

As percentagens de variância explicada para a subescala relativa aos superiores imediatos atingem valores consideráveis, o que igualmente vem confirmar o seu valor psicométrico.

A característica saliente que emerge destes resultados é a qualidade da justiça explicando $63 \%$ da variância comum na amostra de L e $54 \%$ na

Quadro VIII - Solução factorial após rotação Varimax

$\mathrm{L}$

Itens
$\mathrm{T}$

$\begin{array}{lllll} & \text { I } & \text { II } & \text { I } & \text { III }\end{array}$

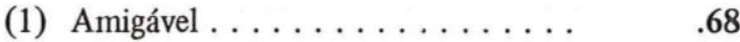

(2) Indelicado . . . . . . . . .

(3) Justo. . . . . . . . . . . .

(4) Competente ............

(5) Sinto que posso discutir problemas com ele . . . . . . . . . . . . .

(6) Quesilento ..............

(7) Organizado. .............

(8) Convencido . . . . . . . . .

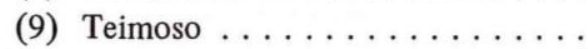

(10) Seguro. ..............

(11) Aceita as minhas sugestões .....

(12) Interfere demais no meu trabalho..

(13) Sei que posso contar com o seu apoio quando tomo uma decisão

.57

.48

.65

.41

$$
.68
$$

.56

.08

.55

.81

$-.26$

.85

.47

.71

\section{.33}

.46

.36

.07

.05

.77

$-.21$

.54

.42

.54

.87

.22

.70

.18

.32

.01

.67

.02

.28

.37

.54

$-.01$

.83

.29

.11

.88

.15

$.63-.01$

,29

.67

.05

.13

$.54 \quad .50 \quad-.15$

.69

.04

L - \% acumulada I $(61,2 \%)$ II $(69,5 \%)$

$\mathrm{T}-\%$ acumulada I $(36,4 \%)$ II $(51,6 \%)$ III $(60,3 \%)$ 
Variáveis

Independentes
Amostra L

\begin{tabular}{lllllllll}
\hline $\mathrm{R}^{2}$ & $\Delta \mathrm{R}^{2}$ & $\mathrm{p}^{*}$ & Beta & se & $\mathrm{B}$ & se & $\mathrm{sr}^{2}$ & $\mathrm{pr}^{2}$
\end{tabular}

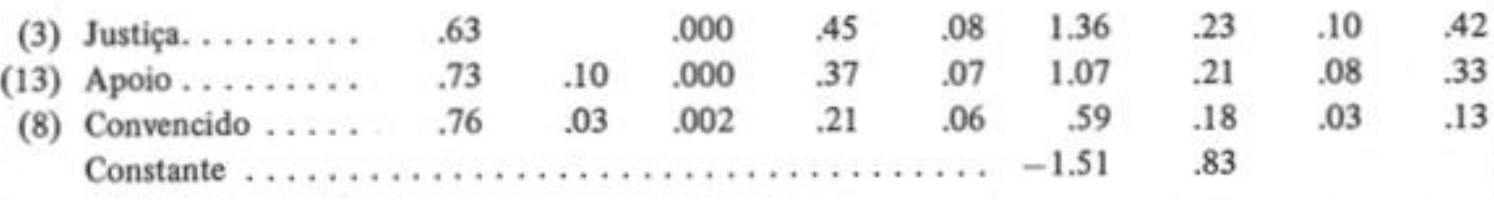

Amostra T

\begin{tabular}{llllllllll}
\hline $\mathrm{R}^{2}$ & $\Delta \mathrm{R}^{2}$ & $\mathrm{p}^{*}$ & Beta & se & $\mathrm{B}$ & $\mathrm{se}$ & $\mathrm{st}^{2}$ & $\mathrm{pr}^{2}$
\end{tabular}

(3) Justiça....... .54

$54 \quad 000$

$\begin{array}{lllll}.37 & .07 & .1 .15 & .24 & .08\end{array}$

(5) Apoio ....... .65

$.11 \quad .000$

.23

.07

.69

.21

.04

.17

(2) Delicadeza ..... $\quad .70$

$.05 \quad .000$

.24

.06

.1 .13

.31

.05

.22

(8) Convencido ..... . .76

$.06 \quad .002$

.22

.07

.50

$.16 \quad .03$

.15

(6) Quesilento..... .77

.01

.033

.16

.07

.41

.17

.02

.10

(13) Apoio ....... .79 .02

$.032 \quad .16$

.07

.47

$.21 \quad .02$

.08

Constante $\ldots \ldots \ldots \ldots \ldots \ldots \ldots$

.032

* - Significância do acréscimo de variância explicada

se - Erro padrão

st - Coeficiente de correlação semiparcial

$\mathrm{pr}$-Coeficiente de correlaçăo parcial

amostra de T. Ê a sua maior ou menor presença o que determina a atitude de maior ou menor aceitaçăo dos chefes pelos subordinados. As características que em seguida melhor discriminem a actuação dos chefes săo as que dizem respeito ao maior ou menor apoio que ele presta aos subordinados nas relaçōes de trabalho e em último lugar, e com peso reduzido, $\hat{e}$ que vêm as qualidades de trato humano reveladas pelos chefes. Note-se que tanto em $\mathrm{L}$ como em $\mathrm{T}$ a atitude para com os superiores é, em média, favorável.

Subescala IV (Colegas do mesmo nível)

Examinaremos primeiramente a estrutura fac- torial da escala. No Quadro X reproduzem-se os resultados obtidos:

Em ambas as amostras a atitude para com os colegas ế favorável, embora mais ainda em $\mathrm{T}(\overline{\mathrm{x}}=6.3)$ do que em $L(\bar{x}=4.9)$. A estrutura factorial desta escala sugere um primeiro factor relacionado com qualidades ligadas ao trabalho e um segundo e terceiro factores ligados a qualidades pessoais.

No que se refere à correlação/regressấo múltipla os resultados estão resumidos no Quadro XI.

Tratando-se em ambos os casos de características positivas há todavia diferenças nos padrōes das duas amostras. Em L os colegas șáo tanto mais apreciados quanto menos maçadores ( $40 \%$ da variảncia explicada) e quanto mais inteligentes 
L

Itens
$\mathrm{T}$

\begin{tabular}{lllll}
\hline I & II & I & II & III
\end{tabular}

\begin{tabular}{|c|c|c|c|c|c|c|}
\hline (1) & Amigáveis $\ldots \ldots \ldots \ldots \ldots \ldots$ & .34 & .65 & .74 & .25 & .08 \\
\hline (2) & Inteligentes $\ldots \ldots \ldots \ldots \ldots$ & .47 & .67 & .23 & .72 & -.11 \\
\hline (3) & Parecidos comigo. . . . . . . . & -.18 & .87 & -.22 & .72 & .27 \\
\hline (4) & Sem experiência $\ldots \ldots \ldots \ldots$ & .48 & .21 & .15 & .38 & .82 \\
\hline (5) & Fora de moda $\ldots \ldots \ldots \ldots \ldots$ & .60 & .45 & .68 & .26 & .26 \\
\hline (6) & $\begin{array}{l}\text { Muitos deles não deviam ter che- } \\
\text { gado onde chegaram. } \ldots \ldots \ldots \ldots\end{array}$ & .52 & .18 & .23 & -.33 & .78 \\
\hline (7) & Incapazes de novas ideias $\ldots \ldots \ldots$ & .85 & .03 & .58 & -.40 & .19 \\
\hline (8) & Convencidos. . . . . . . . . & .74 & .10 & .79 & .14 & -.14 \\
\hline (9) & Maçadores $\ldots \ldots \ldots \ldots \ldots \ldots$ & .46 & .62 & .60 & .19 & .34 \\
\hline
\end{tabular}

L - \% acumulada I $(41,6 \%)$ II $(55,6 \%)$

$\mathrm{T}-\%$ acumulada I $(34,6 \%)$ II $(58,2 \%)$ III $(63,5 \%)$

( $8 \%$ ) enquanto que em T os colegas são sobretudo apreciados em função das características amistosas (39\%) e por não haver conflitos de estatuto (7\%). Os resultados sugerem assim um inter-relacionamento ao nível do grupo informal mais favorável em $\mathrm{T}$ do que em $\mathrm{L}$.

\section{Subescala V (Perspectivas de Promoção)}

No Quadro XII reproduzem-se os resultados da análise factorial da subescala.

A estrutura factorial identificada sugere, no caso da amostra L, que o primeiro factor está associado às expectativas pessoais de promoção e o segundo factor às perspectivas objectivas oferecidas.

Em ambos os casos a atitude dos sujeitos é desfavorável considerando as perspectivas baixas $(\bar{x}=3 \cdot 5)$. Em T a atitude é mais favorável $(\bar{x}=4.9)$ estando o primeiro factor associado às perspectivas objectivas oferecidas pela empresa, o segundo factor às expectativas pessoais, emergindo ainda um terceiro factor diferenciado, saturado apenas pelo item relativo ao tempo de permanência no mesmo nível acrescentando mais $13 \%$ de variância explicada.

No que se refere à correlação/regressão múltipla os resultados estão reunidos no Quadro XIII.

Os resultados obtidos sugerem que em $\mathrm{L}$ as fracas perspectivas de promoção estão primariamente associadas às baixas expectativas pessoais (37\% da variância) e em segundo lugar às perspectivas $o b$ jectivas percepcionadas $(+10 \%)$. Em contrapartida em $\mathrm{T}$ as boas perspectivas estão associadas às saidas que, de acordo com a percepção dos sujeitos, o emprego oferece. A variância explicada, de $13 \%$, é todavia baixa o que sugere grande variabilidade dos sujeitos respondentes e, provavelmente, associação com outras causas de satisfação relativamente à empresa e ao trabalho actual.

\section{Subescala VI (Constante do Trabalho)}

No Quadro XIV reproduzem-se os resultados da análise factorial da subescala VI. 
Variáveis
Independentes
Amostra L

\begin{tabular}{lllllllll}
\hline $\mathrm{R}^{2}$ & $\Delta \mathrm{R}^{2}$ & $\mathrm{p}^{*}$ & Beta & $\mathrm{se}$ & $\mathrm{B}$ & $\mathrm{se}$ & $\mathrm{sr}^{2}$ & $\mathrm{pr}^{2}$
\end{tabular}

$\begin{array}{lllllllll}\text { (9) Maçadores . . . . . } & .40 & .000 & .33 & .09 & .89 & .26 & .07 & .15\end{array}$

$\begin{array}{llllllllll}\text { (2) Inteligentes } \ldots \ldots & .48 & .08 & .000 & .22 & .09 & .64 & .28 & .03 & .06\end{array}$

$\begin{array}{llllllllll}\text { (7) Novas ideias } \ldots \ldots & .52 & .04 & .012 & .21 & .08 & .47 & .19 & .03 & .08\end{array}$

$\begin{array}{lllllllllll}\text { (1) Amigáveis } \ldots \ldots & .5 & .5 & .01 & .020 & .21 & .09 & .61 & .26 & .03 & .07\end{array}$

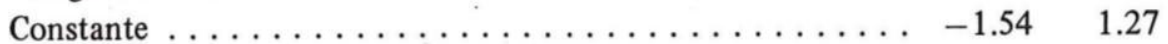

Amostra T

\begin{tabular}{llllllllll}
\hline $\mathrm{R}^{2}$ & $\Delta \mathrm{R}^{2}$ & $\mathrm{p} *$ & Beta & $\mathrm{se}$ & $\mathrm{B}$ & $\mathrm{se}$ & $\mathrm{sr}^{2}$ & $\mathrm{pr}^{2}$ \\
\hline
\end{tabular}

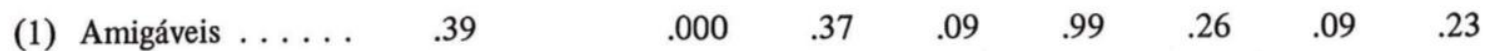

(6) Muitos não deviam ter chegado onde

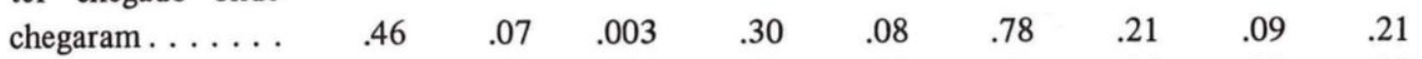

$\begin{array}{llllllllll}\text { (2) Inteligentes } \ldots \ldots & .54 & .08 & .001 & .17 & .09 & .47 & .25 & .02 & .05\end{array}$

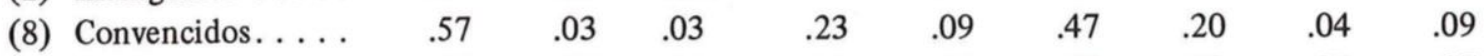

$\begin{array}{llllllllll}\text { (3) Parecidos comigo. . } & .60 & .03 & .02 & .19 & .09 & .40 & .20 & .03 & .07\end{array}$

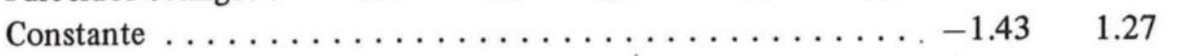

* - Significância do acréscimo de variância explicada

se - Erro padrão

sr - Coeficiente de correlação semiparcial

pr - Coeficiente de correlação parcial

A atitude total para com o conteúdo do trabalho é favorável tanto em $\mathrm{L}\left(\overline{\mathrm{x}}={ }^{4-8}\right) \int \therefore \sqrt{ } \therefore{ }^{\circ} \sqrt{ } \nabla$

A atitude total para com o conteúdo do trabalho é favorável tanto em $\mathrm{L}(\overline{\mathrm{x}}=4.8)$ como em $\mathrm{T}$ $(\bar{x}=6.1)$ embora significativamente maior em $T$. A estrutura factorial da amostra de L sugere em primeiro factor geral de valorização do conteúdo do trabalho, um segundo factor associado ao prestigio social que a função confere e um terceiro factor associado ao par responsabilidade/autoridade. Em T verifica-se, mais uma vez, uma estrutura mais diversificada e complexa em que o primeiro factor está associado à satisfação pessoal com o conteúdo do trabalho, o segundo factor à valorização pessoal, o terceiro ao prestígio, o quarto à autoridade e o quinto à experiência.

No que se refere à análise da correlação/regressão múltipla os resultados são resumidos no Quadro XV.

De acordo com os resultado obtidos é a estimulação a principal característica associada à atitude favorável para com o conteúdo do trabalho, tanto em L (39\%) como em T (31\%). Em segundo lugar a população de $\mathrm{L}$ atribui importância ao carácter não fatigante e à utilidade do trabalho, $(=15 \%)$ enquanto que a população de $\mathrm{T}$ atribui maior importância à experiência exigida $(=10 \%)$ 
Itens

$\mathrm{L}$

$\overline{\text { I II }}$

$\mathrm{T}$

II III
(1) Boas oportunidades . . . . . . . . . . $\quad .27$

(2) Boas perspectivas

(3) Espero chegar mais acima . . . . . .

(4) Política de promoções justas . . . .

(5) Emprego sem saída . . . . . . . .

(6) Experiência aumenta perspectivas. .

(7) Há muito tempo ao mesmo nível ..

(8) Qualificações e treino aumentam as perspectivas ...........
$.27 \quad .65$

$\begin{array}{ll}.13 & .78\end{array}$

$.69 \quad .18$

$.21 \quad .42$

$\begin{array}{ll}.70 & .17\end{array}$

$.91 \quad .16$

$.02 \quad .66$

$.89 \quad .18$ $\begin{array}{rrr}.81 & -.02 & -.15 \\ .80 & .20 & .17\end{array}$

$.04 \quad .82$

$\begin{array}{lrr}.60 & .50 \quad .06\end{array}$

$\begin{array}{lll}.60 & -.07 & .42\end{array}$

$\begin{array}{lll}.21 & .61 & .36\end{array}$

$\begin{array}{lll}.04 & .07 & .90\end{array}$

$.01 \quad .80$

$\mathrm{L}-\%$ acumulada I $(41,0 \%)$ II $(56 \%)$

$\mathrm{T}-\%$ acumulada I $(34,1 \%)$ II $(52,4 \%)$ III $(65,5 \%)$

Quadro XIII - Correlação/Regressão Múltipla da Subescala V relativamente à Subescala de Controlo V (VD)

\begin{tabular}{|c|c|c|c|c|c|c|c|c|c|c|}
\hline \multirow{2}{*}{\multicolumn{2}{|c|}{$\begin{array}{c}\text { Variáveis } \\
\text { Independentes }\end{array}$}} & & \multicolumn{3}{|c|}{ Amostra L } & \multirow[b]{2}{*}{ se } & \multirow[b]{2}{*}{$\mathrm{sr}^{2}$} & \\
\hline & & $\mathrm{R}^{2}$ & $\Delta \mathrm{R}^{2}$ & $\mathrm{p}^{*}$ & Beta & se & B & & & \\
\hline (8) & Qualif. e treino ... & .29 & & .000 & .29 & .09 & .59 & .20 & .06 & .11 \\
\hline (5) & Saídas......... & .37 & .08 & .001 & .29 & .09 & .60 & .20 & .06 & .11 \\
\hline (7) & Tempo no nível. . . & .43 & .06 & .006 & .22 & .08 & .46 & .17 & .05 & .09 \\
\hline (1) & Oportunidades ... & .47 & .04 & .018 & .21 & .08 & .56 & .23 & .04 & .07 \\
\hline & Constante ...... & $\ldots$ & $\ldots$ & $\ldots$ & $\ldots$ & $\ldots$ & -.39 & .76 & & \\
\hline
\end{tabular}

Amostra T

\begin{tabular}{lllllllll}
\hline $\mathrm{R}^{2}$ & $\Delta \mathrm{R}^{2}$ & $\mathrm{p} *$ & Beta & se & $\mathrm{B}$ & se & $\mathrm{sr}^{2}$ & $\mathrm{pr}^{2}$ \\
\hline
\end{tabular}

$\begin{array}{llllllll}\text { (5) Saída } \ldots \ldots \ldots \ldots & .13 & .13 & .013 & .36 & .11 & .76 & .24\end{array}$

Constante . . . . . . . . 59

* _ Significância do acréscimo de variância explicada

se - Erro padrão sr - Coeficiente de correlação semiparcial $\mathrm{pr}$-Coeficiente de correlação parcial 


\begin{tabular}{|c|c|c|c|c|c|c|c|c|c|}
\hline \multirow{2}{*}{\multicolumn{2}{|c|}{ Itens }} & \multicolumn{3}{|c|}{$\mathrm{L}$} & \multicolumn{5}{|c|}{$\mathrm{T}$} \\
\hline & & I & II & III & I & II & III & IV & V \\
\hline (1) & Frustrante $\ldots \ldots \ldots$ & .84 & .07 & .27 & & & .83 & & \\
\hline (2) & Respeitado. . . . . . . . & .60 & .22 & .23 & & & .60 & & \\
\hline (3) & Desencorajante ...... & .78 & .12 & .41 & .57 & & & & \\
\hline (4) & Dá satisfação . . . . . . . . & .79 & .24 & .19 & .73 & & & & \\
\hline (5) & Responsabilidade. . . . . . & .10 & .17 & .71 & & .55 & & & \\
\hline (6) & Respeito por si próprio. . & .50 & .56 & .08 & .73 & & & & \\
\hline (7) & Qualificações . . . . . . . & & & & & & & .74 & \\
\hline (8) & Adequação. . . . . . . . . . & .73 & .16 & -.20 & .64 & & .58 & & \\
\hline$(9)$ & O tempo passa depressa & .72 & -.08 & .10 & & & & & \\
\hline (10) & Utilidade . . . . . . . . . & .30 & .63 & .13 & .65 & & & & \\
\hline (11) & Autoridade. . . . . . . . & .19 & -.11 & .80 & & & & .67 & \\
\hline (12) & Experiência $\ldots \ldots \ldots$ & -.01 & .78 & .05 & & & & & .77 \\
\hline (13) & Aprendizagem. . . . . . & .73 & .47 & -.02 & & .71 & & & \\
\hline (14) & Estimulante . . . . . . & .79 & .24 & .17 & & .80 & & & \\
\hline
\end{tabular}

associação esta que se afigura menos clara.

\section{Subescala VII (Subordinados)}

Finalmente analisou-se ainda a subescala VII, até agora ainda não cruzada com as restantes subescalas por virtude de ter sido preenchida por um escasso número de sujeitos, ou seja, pelos que exercem funções de chefia e têm subordinados sob o seu controlo. Dada a exiguidade das amostras haverá que manter reservas quanto aos resultados obtidos. Na medida porém em que se atingem, também aqui, níveis de significância estatísticas julga-se que tais resultados serão de algum modo a tais a título indicativo.

Como para os casos anteriores procedemos em primeiro lugar a uma análise factorial da subescala mas reunindo as duas amostras. Os resultados estão resumidos no Quadro XVI.
Tanto em L $(\mathrm{N}=28)$ como em $\mathrm{T}(\mathrm{N}=8)$ a atitude para com os subordinados é favorável. A média da subescala global para L é $\bar{x}=5.5(\mathrm{Dp}=1.7)$ e para $\mathrm{T} \overline{\mathrm{x}}=6.25(\mathrm{DP}=1.5)$ não sendo a diferença significativa .

A estrutura obtida sugere que o primeiro factor é um índice geral de satisfação para com os subordinados, que o segundo factor diz respeito às qualidades pessoais (inteligência, aplicação de confiança e responsáveis) e o terceiro factor diz respeito à sua adequação às funções que exercem.

Seguidamente procedeu-se a uma análise de correlação/regressão múltipla tanto para a amostra conjunta como em separado para L e T. Os resultados acham-se resumidos no Quadro XVII.

Com base nestes resultados emerge com saliência a importância do factor confiança, nos subordinados para determinar uma atitude favorável da parte dos chefes, em L, enquanto que em T é sobretudo a não necessidade de controlo que mais as- 


\section{Quadro XV - Correlação/Regressão Múltipla da Subescala VI relativamente à Subescala de Controlo VI (VD)}

Variáveis

Independentes

Amostra L

\begin{tabular}{|c|c|c|c|c|c|c|c|c|}
\hline $\mathrm{R}^{2}$ & $\Delta \mathrm{R}^{2}$ & $\mathrm{p} *$ & Beta & se & B & se & $\mathrm{sr}^{2}$ & $\mathrm{pr}^{2}$ \\
\hline .39 & & .000 & .39 & .09 & .86 & .19 & .11 & .24 \\
\hline .47 & .08 & .001 & .30 & .08 & .65 & .18 & .08 & .17 \\
\hline .54 & .07 & .000 & .30 & .08 & .94 & .26 & .08 & .17 \\
\hline . & & & & $\ldots$ & -.61 & .90 & & \\
\hline
\end{tabular}

Amostra T

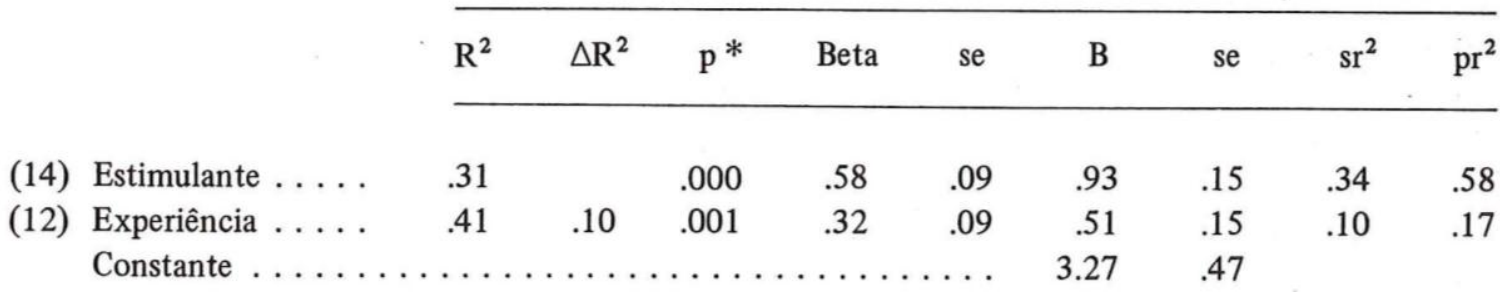

* - Significância do acréscimo de variância explicada se - Erro padrão

sociada se acha à aceitação dos subordinados. Esta diferença pode ser uma indicação de diferentes estilos de chefia, mais concretizada e controlada em L e mais permissiva em $T$.

\section{CONCLUSÕES}

A aplicação do inquérito de satisfação no trabalho a dois serviços de natureza idêntica pertencentes à mesma organização geograficamente distantes permite tirar as seguintes conclusões.

Em termos muito globais a amostra $\mathrm{T}$ tem uma atitude significativamente mais favorávem em todos os aspectos com excepção da atitude dos chefes para com os subordinados onde não se encontraram diferenças.

Em L observa-se insatisfação relativamente à organização como um todo, às remunerações e às perspectivas de promoção e satisfação moderada sr - Coeficiente de correlação semiparcial

$\mathrm{pr}$ - Coeficiente de correlação parcial

relativamente aos superiores imediatos, às relações com os colegas do mesmo nível e ao conteúdo do trabalho.

Em T os níveis de satisfação são elevados em todos os aspectos sendo os menos elevados os respeitantes a perspectivas de promoção e remunerações.

Em termos de pesos relativos dos factores enunciados verificou-se que em $\mathrm{L}$ o peso dos factores de insatisfação, ou seja, das fracas perspectivas de promoção, é duas vezes superior ao peso da principal causa de satisfação detectada, a saber, a impressão favorável relativamente às chefias. Em contrapartida em $\mathrm{T}$ a principal causa de satisfação reside na impressão favorável relativamente às chefias e, em menor grau, ao tipo de trabalho efectuado.

Especificando ainda mais a análise relativamente a estes factores mais explicativos verificou-se que, em L, as perspectivas de promoção se acham primariamente associadas às baixas expectativas pessoais e em segundo lugar âs perspectivas objectivas percepcionadas. Por seu turno a satisfa- 
Itens

Factores

\begin{tabular}{lll}
\hline I II & III
\end{tabular}

(1) Pouco inteligentes $\ldots \ldots \ldots \ldots \ldots \ldots \ldots \ldots$ .54

(2) Preguiçosos .80

(3) Preferível mais velhos. . . . . . . . . . . .

(4) Trabalham bem como grupo $\ldots \ldots \ldots \ldots \ldots$

.80

(5) Não são de confiança . . . . . . . . . . . . .84

(6) Pouco treinados $\ldots \ldots \ldots \ldots \ldots \ldots \ldots \ldots$

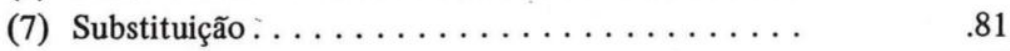

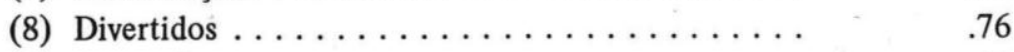

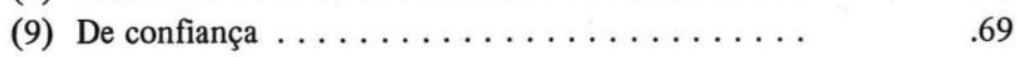

(10) Necessário controlar. . . . . . . . . . . . . .

(11) Competentes $\ldots \ldots \ldots \ldots \ldots \ldots \ldots \ldots$

.65

\% Variância acumulada I (43,2 \%) II (57,3 \%) III $(66,6 \%)$

Quadro XVII - Correlação/Regressão Múltipla da Subescala VII relativamente à Subescala de Controlo

\begin{tabular}{|c|c|c|c|c|c|c|c|c|c|c|}
\hline \multirow{2}{*}{\multicolumn{2}{|c|}{$\begin{array}{c}\text { Variáveis } \\
\text { Independentes }\end{array}$}} & \multicolumn{9}{|c|}{ Ámostra $(\mathrm{L}+\mathrm{T})$} \\
\hline & & $\mathrm{R}^{2}$ & $\Delta \mathrm{R}^{2}$ & $\mathrm{p} *$ & Beta & se & B & se & $\mathrm{sr}^{2}$ & $\mathrm{pr}^{2}$ \\
\hline \multirow[t]{3}{*}{ (9) } & Confiança $\ldots . .$. & .76 & & .000 & .87 & & & & & \\
\hline & & \multicolumn{9}{|c|}{ Amostra L } \\
\hline & & $\mathrm{R}^{2}$ & $\Delta \mathrm{R}^{2}$ & $\mathrm{p} *$ & Beta & se & B & se & $\mathrm{sr}^{2}$ & $\mathrm{pr}^{2}$ \\
\hline (9) & Confiança ...... & .78 & & .000 & .85 & .10 & 2.19 & .27 & .71 & 1.0 \\
\hline \multirow{4}{*}{ (6) } & Treino. . . . . . . & .82 & .04 & .047 & .22 & .10 & .40 & .19 & .05 & .27 \\
\hline & Constante $\ldots \ldots$ & $\ldots$ & $\ldots$ & $\ldots \ldots$ & $\ldots \ldots$ & $\ldots$ & -.32 & .78 & & \\
\hline & & \multicolumn{9}{|c|}{ Amostra $\mathrm{T}$} \\
\hline & & $\mathrm{R}^{2}$ & $\Delta \mathrm{R}^{2}$ & $\mathrm{p} *$ & Beta & se & B & se & $\mathrm{sr}^{2}$ & $\mathrm{pr}^{2}$ \\
\hline \multirow[t]{2}{*}{ (10) } & Controlo . . . . . . & .71 & & .008 & .85 & $.20^{\circ}$ & 2.5 & .65 & & \\
\hline & Constante ..... & $\ldots$ & $\ldots$ & $\ldots$ & $\ldots$ & $\ldots$ & .00 & 1.75 & & \\
\hline
\end{tabular}

* - Significância do acréscimo de variância explicada se--Erro padrão sr - Coeficiente de correlação semiparcial

$\mathrm{pr}$ - Coeficiente de correlação parcial 
ção, ainda que moderada, face ao comportamento das chefias se deve antes de mais à percepção duma actuação justa e, em segundo lugar, ao apoio profissional proporcionado.

Em T verificou-se, por seu turno, que a atitude favorável relativamente às chefias, causa principal da satisfação global, se deve igualmente à percepção duma actuação justa, ao apoio por eles prestado e também às qualidades de trato percepcionadas. Quanto à satisfação derivada do conteúdo do trabalho deverá atribuir-se antes de mais a que o pessoal se encontra em início de carreira pelo que as tarefas, embora repetitivas como é o caso do registo de dados, são aceites favoravelmente.

A extrair alguma conclusão global da análise efectuada ela residiria na importância do efeito liderança enquanto controlo organizacional.

No caso dum serviço como o de $\mathrm{L}$ onde só existe frustração e descontentamento tanto no que se refere a perspectivas de carreira, como a vencimentos auferidos, os chefes exercem uma acção moderadora atenuando os efeitos negativos. No caso de $\mathrm{T}$ a aç̧ão das chefias terá porventura efeitos mais motivacionais contribuindo decisiva-

\section{REFERENCIAS}

P. C. SMITH, L. M. KENDALL e C. L. HULIN (1969) - The measurement of satisfacion in work and retirement. Chicago: Rand McNally.

P. B. WARR e T. ROUTLEDGE (1969) - «An opinion scale for the study of Manager's Job Satisfaction"-Occupational Psychology, 43: 95-109. mente para o elevado moral expresso nas respostas ao inquérito. Igualmente de assinalar como conclusão a reter que, sendo tão central o efeito de liderança, o aspecto que por seu turno dele emerge como mais saliente é a justiça de actuação. A análise não permite ir aqui mais longe mas será importante, em investigações futuras, aprofundar e operacionalizar este conceito de justiça por forma a determinar a estrutura multidimensional do seu espaço semântico.

\section{SUMMARY}

In the present work, the authors proceed to the psychometric analysis of the properties of a Job Satisfaction inventory (developed by Warr $\&$ Routledge, 1969), in its portuguese adaptation. An intensive statistical study of the external and construct validity, as well as of the internal consistency of the inventory and of its sevens subscales is perfurmed, after its applications to two examples of workers in a national computer service.

D. T. CAMPBELL e D. W. FISKE (1959) - «Convergent and discriminant validation by the multitrait-multimethod matrix». Psychological Bulletin, 56: 81-105.

N. H. NYE e C. H. HULL (1979)-The SCSS preliminary User's Manuel. SPSS Inc., N. Y.

J. COHEN e P. COHEN (1975) - Applied Multiple Regression/Correlation Analysis for the Behavioral Sciences. Wiley e Sons. 


\section{ANEXO}

Apresenta-se a seguir o questionário na sua forma final. Atribui-se uma pontuação de 3 nos itens positivos à resposta $\mathrm{S}(\mathrm{Sim})$ e nos itens negativos à resposta $\mathrm{N}$ (Não). Entre parêntesis são indicadas as correlações de cada item com o score total obtido na subescala primeiro para a amostra $\mathrm{L}(\mathrm{N}=86)$ e em segundo lugar para a amostra $\mathrm{T}(\mathrm{N}=68)$.

\section{QUESTIONÁRIO SOBRE SATISFAÇÃO NO TRABALHO}

I - As características a seguir dizem respeito à organização como um todo. Assinale com um círculo, em cada uma delas, a resposta mais aplicável.

$\mathrm{S}$ ?N Boa reputação $(.170, .146)$

S?N Precisaria de sangue novo na Direcção $(.503, .533)$

S?N A Direcção faz segredo de coisas que deveríamos conhecer $(.326, .250)$

$\mathrm{S} ? \mathrm{~N}$ Eficiente $(.376, .319)$

$\mathrm{S}$ ?N Distinções muito grandes entre categorias $(.431, .342)$

$\mathrm{S}$ ?N Excesso de regras e regulamentos $(.472, .292)$

S?N Coordenação insuficiente entre departamentos $(.573, .213)$

S?N Merecedora do nosso esforço (.499, .308)

II - As características a seguir aplicam-se a remunerações. Por cada uma assinale com um círculo conforme apropriado.

S?N Razoável (.503, .515)

S?N Pouco para aquilo que faço $(.463, .515)$

S?N Do mesmo nível que os colegas da mesma idade desempenhando funções semelhantes $(.085, .462)$

S?N O seu nível de vida é tão bom como o dos amigos com quem convive socialmente $(.300, .318)$

III - As características a seguir aplicam-se ao seu superior imediato. Para cada uma assinale com um círculo conforme apropriado.
S?N Amigável $(.858,565)$

$\mathrm{S}$ ?N Indelicado $(.833, .366)$

S?N Justo $(.798, .662)$

S?N Competente $(.837, .561)$

S?N Sinto que posso discutir problemas com ele $(.728, .608)$

S?N Quesilento $(.793, .311)$

S?N Organizado $(.612, .589)$

S?N Convencido $(.644, .530)$

S?N Teimoso $(.622, .567)$

S?N Seguro $(.655, .435)$

$\mathrm{S} ? \mathrm{~N}$ Aceita as minhas sugestões $(.687, .410)$

$\mathrm{S}$ ?N Interfere demais no meu trabalho $(.745, .419)$

S?N Sei que posso contar com o seu apoio quando tomo uma decisão $(.735, .432)$

IV - Os enunciados a seguir aplicam-se aos colegas de seu nivel. Para cada um assinale com um círculo conforme apropriado.

S?N Amigáveis $(.568, .563)$

$\mathrm{S}$ ?N Inteligentes $(.676, .299)$

S?N Muito parecidos comigo $(.285, .418)$

S?N Sem experiência suficiente $(.400, .368)$

S?N Muito fora de moda $(.623, .608)$

S?N Muitos deles não deviam ter chegado onde chegaram $(.392, .276)$

S?N Incapazes de novas ideias $(.539, .286)$

$\mathrm{S}$ ?N Convencidos $(.505, .464)$

$\mathrm{S} ? \mathrm{~N}$ Maçadores $(.622, .550)$

$\mathrm{V}$ - Os enunciados a seguir aplicam-se a perspectivas de promoção. Para cada um assinale com um círculo conforme apropriado.

$\mathrm{S} ? \mathrm{~N}$ Boas oportunidades $(.439, .351)$

$\mathrm{S}$ ?N Perspectivas tão boas como em qualquer outro serviço $(.406, .578)$

$\mathrm{S}$ ?N Espero chegar mais acima $(.536, .257)$

$\mathrm{S}$ ?N A política de promoção é pouco justa $(.291, .580)$

S?N Emprego sem saída (.520, .376)

$\mathrm{S}$ ?N A minha experiência aumenta as minhas perspectivas $(.707, .456)$

S?N Acho que já estou há muito tempo no mesmo nível $(.265, .456)$

$\mathrm{S}$ ?N As minhas qualificações e treino aumentam as minhas perspectivas $(.721$, .372) 
VI - Os enunciados a seguir aplicam-se ao trabalho em si mesmo. Para cada um assinale com um círculo conforme apropriado.

S?N Frustrante $(.754, .229)$

S?N Respeitado $(.604, .326)$

S?N Desencorajante $(.780, .464)$

S?N Dá satisfação $(.760, .568)$

S?N Responsabilidade insuficiente (.336, .286)

S?N Proporciona sentimento de respeito por si próprio $(.603, .474)$

$\mathrm{S}$ ?N Não requer qualificações específicas (.320, .107)

S?N Tipo de trabalho pouco apropriado para mim $(.537, .498)$

S?N O tempo passa depressa $(.543, .348)$

S?N Útil $(.477, .240)$

S?N Disponho de pouca autoridade (.335, .230)
S?N Exige muita experiência $(.235, .164)$

S?N Posso aprender muito com este trabalho $(.735, .318)$

S?N Estimulante $(.752, .474)$

VII-Os enunciados a seguir aplicam-se aos subordinados. Para cada um assinale conforme apropriado.

S?N Pouco inteligente

S?N Preguiçoso

S?N Seria preferível ter homens mais velhos

S?N Trabalham bem como grupo

S?N Não se pode confiar neles

S?N Pouco treinados

S?N Se possível substituía a maior parte deles

S?N Divertidos

S?N De confiança

S?N É necessário controlá-los de perto

S?N Competentes 